\title{
G-CSF: One of The Vehicles for Communication Between Trophoblasts and Macrophages, Which May Cause Problems in Recurrent Spontaneous Abortion
}

\section{Peng Gao}

Tongji Hospital of Tongji Medical College of Huazhong University of Science and Technology Haiyi Liu

Tongji Hospital of Tongji Medical College of Huazhong University of Science and Technology Ying Zha

Tongji Hospital of Tongji Medical College of Huazhong University of Science and Technology Lijie Wei

Tongji Hospital of Tongji Medical College of Huazhong University of Science and Technology Xuan Zhou

Tongji Hospital of Tongji Medical College of Huazhong University of Science and Technology Shenglan Zhu

Tongji Hospital of Tongji Medical College of Huazhong University of Science and Technology Huiting Zhang

Tongji Hospital of Tongji Medical College of Huazhong University of Science and Technology

\section{Xuan Gao}

Tongji Hospital of Tongji Medical College of Huazhong University of Science and Technology

\section{Yi Jiang}

Tongji Hospital of Tongji Medical College of Huazhong University of Science and Technology

\section{YuTing Chen}

Tongji Hospital of Tongji Medical College of Huazhong University of Science and Technology Jiaqi Li

Tongji Hospital of Tongji Medical College of Huazhong University of Science and Technology Jingyi Zhang

Tongji Hospital of Tongji Medical College of Huazhong University of Science and Technology Jun $\mathrm{Yu}$

Tongji Hospital of Tongji Medical College of Huazhong University of Science and Technology Shaoshuai Wang

Tongji Hospital of Tongji Medical College of Huazhong University of Science and Technology Ling Feng ( $\sim$ fltj007@163.com)

Tongji Hospital of Tongji Medical College of Huazhong University of Science and Technology 


\section{Research}

Keywords: recurrent spontaneous abortion, trophoblast, macrophage, G-CSF, inflammation

Posted Date: August 31st, 2021

DOl: https://doi.org/10.21203/rs.3.rs-824254/v1

License: (c) (1) This work is licensed under a Creative Commons Attribution 4.0 International License. Read Full License

Version of Record: A version of this preprint was published at Placenta on March 1st, 2022. See the published version at https://doi.org/10.1016/j.placenta.2022.03.125. 


\section{Abstract \\ Background}

The etiology of about half of the patients with recurrent spontaneous abortion (RSA) remains unclear, and the imbalance of the immune inflammatory response at the mother-foetal interface may be one of the keys to the onset. Granulocyte-colony stimulating factor (G-CSF) is thought to have a protective effect on pregnancy, and its absence may lead to pregnancy failure, but the evidence is scant. This study aims at investigating whether the loss of G-CSF induced RSA by affecting cell communication at the maternalfoetal interface.

\section{Results}

G-CSF was mainly expressed in villus rather than decidua, and the expression in RSA tissues was lower than that in normal tissues. Down-regulation of G-CSF in trophoblasts resulted in decreased cell activity. Trophoblast-derived exosomes inhibited macrophage activation, while G-CSF free exosomes did not. Intraperitoneal injection of G-CSF improved the pregnancy outcome of RSA mice, and the expression of GCSF and its receptor at the mother-foetal interface also changed.

\section{Conclusion}

The expression of G-CSF was found to be decreased in villi of patients with RSA. The absence of G-CSF weakens the immune suppression of trophoblasts against macrophages, and the function of trophoblasts is impaired, which may be a key factor in the occurrence of RSA. G-CSF decreased the rate of abortion in RSA mice, and might provide some assistance in the treatment of patients with RSA.

\section{Background}

Recurrent spontaneous abortion (RSA) is defined as two or more consecutive spontaneous abortions with the same sexual partner, with an incidence of about 5-10\% [1-3]. Among them, approximately half of cases remain unexplained $[4,5]$. Our previous studies suggested that the communication between trophoblasts and macrophages was deviated at the maternal-foetal interface of patients with RSA, and the overexpression of NLRP3 inflammasomes triggered abnormal inflammatory response and destroyed the balance between pro-inflammatory and anti-inflammatory states at the maternal-foetal interface [6].

Proper communication between the foetal trophoblast and the immune cells present at the maternalfoetal interface including macrophages is essential for a successful pregnancy. Trophoblasts exert vital roles in early embryo implantation and pregnancy. Trophoblasts invade the endometrium to establish maternal-foetal connection and promote the formation of maternal immune tolerance to the foetus [7]. Inadequate migration of trophoblasts usually leads to failure of maternal-foetal linkage and RSA [8,9]. 
Meanwhile, increasing evidences support that the polarization of decidual macrophages is influenced by trophoblasts [10-13]. Decidual macrophages have considerable plasticity and can effectively respond to environmental signals. The macrophage phenotypes can be roughly divided into pro-inflammatory M1 type and anti-inflammatory $\mathrm{M} 2$ type. Decidual macrophages are close to the $\mathrm{M} 2$ type, and may rapidly transform into M1 type with the progress of pregnancy and environmental changes [11].

Granulocyte-colony stimulating factor (G-CSF), a kind of secretory cytokine closely related to cell proliferation, invasion and differentiation, can be expressed in trophoblasts and endometrium. G-CSF has a positive effect on trophoblast growth, embryo implantation and placental metabolism $[14,15]$. The function of G-CSF is mainly realized by binding to the G-CSF receptor (G-CSFR) on the surface of target cells, and G-CSFR exists in various types of myeloproliferative tissues and cells, such as monocytes macrophages $[16,17]$. Based on the above, G-CSF may be one of the media for the dialogue between trophoblasts and decidual macrophages. Researches in animal models have shown that the loss of GCSF may induce early abortion [16]. Some studies report that in vitro injection of human recombinant GCSF to patients with RSA may improve the pregnancy outcome, and the efficacy is even better than that of clinical commonly used drugs such as low molecular weight heparin and immunoglobulin, while others hold the opposite view [17-19]. This is a problem needs further study.

In the last century, it was discovered that trophoblast vesicles might be shed from the placenta into the mother's peripheral circulation and could be isolated from the mother's peripheral blood as early as 6 weeks of gestation [20,21]. In recent years, exosomes, a clearer concept than "vesicles", have gained increasing attention. Exosomes are membrane-bound vesicles, which exist in almost all biological fluids. Exosomes are rich in protein and RNA and have a strong function in information exchange between cells [22]. Trophoblast-derived exosomes are thought to have the ability to regulate the cytokine profile of recipient cells, such as increased IL-2 expression and decreased CD3 expression in T cells. Literatures support that during implantation and the initial trimester, trophoblast-derived exosomes are capable of recruiting and promoting differentiation of monocytes into a trophoblast-supportive tissue macrophages, which in turn secrete cytokines and chemokines required for trophoblast growth and survival [23]. As a soluble cytokine, G-CSF can be released extracellular or transported by extracellular vesicles such as exosomes to participate in the activation of cell signalling pathways in a paracrine form [24]. Therefore, our purpose is to study whether G-CSF carried by exosomes is involved in the trophoblast-macrophage dialogue, and whether the absence of G-CSF is a key factor leading to the occurrence of RSA.

\section{Results}

\section{Expression of G-CSF and its receptor at maternal-foetal interface in early pregnancy}

The presence of G-CSF and G-CSFR in villous and decidual tissues was detected by RTPCR, western blot and immunohistochemistry. G-CSF was mainly expressed in villi, and the expression in RSA villi was 
significantly lower than that in normal villi. G-CSFR showed the opposite trend, and its expression in RSA decidua was slightly higher than that in normal decidua (Fig. 1 and Additional file 1: figure S1).

\section{Effect of G-CSF on the migration and proliferation of trophoblasts}

Three siRNAs targeting G-CSF were constructed and RTPCR detection showed that all the three siRNAs had silencing effects. The most effective siRNA was selected for subsequent experiments. Forty-eight hours after siRNA transfection, the cells were assayed by transwell and CCK8 tests. The absence of GCSF significantly reduced the migration and proliferation of trophoblasts (Fig. 2 and Additional file 2: figure S2).

\section{Role of G-CSF and exosomes in trophoblasts regulation on macrophages}

The supernatant of trophoblasts after siRNA transfection and normal trophoblasts were collected, exosomes were extracted and added into macrophages medium at a concentration of $100 \mu \mathrm{g} / \mathrm{mL}$ [23]. Meanwhile, recombinant G-CSF was added into the macrophages medium at the concentration of 10,50 , 100, 200 and 500ng/mL, respectively. G-CSF was found to have the most obvious anti-inflammatory effect on macrophages at a concentration of $100 \mathrm{ng} / \mathrm{ml}$, and this concentration was used in subsequent experiments. The morphology, size and specific markers of exosomes were identified by transmission electron microscopy (TEM), nanoparticle tracking analysis (NTA) and western blot (Fig. 3A). The particle size of exosomes was $134.70 \pm 48.20 \mathrm{~nm}$. Since the amplification of trophoblast took about a week, and the effect of siRNA was not permanent, the expression of G-CSF in cells after transfection for 7 days was tested and it was still suppressed. At the same time, the detection of G-CSF in exosomes also showed the silencing effect of transfection (Fig. 3B and Additional file 3: figure S3). Under the laser confocal microscope, the PKH26 labelled exosomes were clearly engulfed by macrophages and surrounded the nucleus. It could be seen at low magnification that the exosomes derived from trophoblasts treated with G-CSF siRNA were more phagocytosed by macrophages than exosomes derived from normal trophoblasts (Fig. 3C). After the phagocytosis of exosomes, the functional activity of macrophages also changed. Normal trophoblasts downregulated the expression of iNOS and TNF-a and upregulated the expression of CD206 and Arg1 in macrophages through exosomes. NLRP3 inflammasome, a molecule thought to play a pro-inflammatory role at the maternal-foetal interface in patients with RSA [6], was also found to be inhibited by trophoblast cell-derived exosomes. These results indicated that trophoblasts could induce the transformation of macrophages to anti-inflammatory M2 type through exosomes. In addition, trophoblast cell-derived exosomes also had inhibitory effects on the migration and proliferation of macrophages. The addition of exogenous G-CSF had the same effects on the macrophages. However, after silencing the G-CSF in the trophoblasts, the above effects disappeared (Fig. 3D and Additional file 4: figure S4). This suggested that G-CSF played a key role in the process of trophoblasts regulating macrophages.

\section{Application of G-CSF in RSA mouse model}


Compared with the Normal group (5.71\%), the abortion rate in the RSA group was $26.96 \%$, indicating the success of the model. The abortion rate was reduced to $10.25 \%$ after intraperitoneal injection of G-CSF, as we expected. Decreased expression of G-CSF was observed in the placentas of RSA mice. The increase of G-CSF in the TREAT group suggested that intraperitoneal injection of G-CSF played a role in the placentas. The expression of G-CSFR in the placentas of the three groups was not statistically different. The expression of NLRP3 inflammasome was increased in the placentas of RSA mice and was inhibited by exogenous G-CSF, which was consistent with the results of cell experiments (Fig. 4 and Additional file 5: figure S5).

\section{Discussion}

The occurrence of RSA is closely related to the imbalance of pro-inflammatory and anti-inflammatory state and the formation barriers of immune tolerance at the maternal-foetal interface, and the disorder of trophoblast-macrophage dialogue is likely to be a crucial part to induce the imbalance $[10,25,26]$. Previous studies have reported that G-CSF plays an important role in establishing immune tolerance between host tissue and donor-derived immune cells [27]. Therefore, we investigated whether G-CSF played the same role at the maternal-foetal interface.

First, tissue samples from the patients were examined. The results showed that G-CSF was mainly expressed in villous tissues, and was lower in RSA samples than in normal tissues, which initially suggested the association between G-CSF and RSA. On the contrary, G-CSFR was more expressed in decidua tissues, and the expression in RSA decidua was slightly higher than that in normal decidua. We hypothesized that the increased expression of G-CSFR in the RSA decidua might be a compensatory response to the decreased G-CSF. After down-regulation of G-CSF, the migration and proliferation of trophoblasts decreased, suggesting that G-CSF did play a role in the growth of trophoblasts. Therefore, the decline in G-CSF levels and the consequent impairment of trophoblast function may be the key factors in the induction of RSA.

The pathogenesis of RSA is not single, and the role of G-CSF at the maternal-foetal interface is not only to regulate the function of trophoblast cells. In order to investigate the role of macrophages in the pathogenesis of RSA, we co-cultured macrophages with trophoblast-derived exosomes. As expected, exosomes without G-CSF no longer inhibited macrophage activity, leading to more M1-type differentiation of macrophages and increased NLRP3 inflammasomes. The association between the overexpression of NLRP3 inflammasomes and RSA had been confirmed, which was consistent with our experimental results $[6,28]$. Therefore, we believe that the loss of G-CSF, on the one hand, disrupts the function of trophoblasts, and on the other hand, interferes with the pro-inflammatory and anti-inflammatory balance at the mother-foetal interface through macrophages, which in turn leads to impaired trophoblasts function $[7,29]$ and ultimately induces RSA.

As previously noted, the jury is still out on the efficacy of G-CSF in treating RSA and more evidence is needed. A number of studies have shown that G-CSF improves endometrial thickness, ovarian follicular 
function and oocyte quality, which may facilitate embryo implantation [30-32]. In our study, intraperitoneal injection of G-CSF into RSA mouse models showed a significant reduction in the abortion rate and inhibition of NLRP3 inflammasomes overexpression at the maternal-foetal interface. Therefore, we can expect that G-CSF may improve pregnancy success by improving the inflammatory state of the mother-foetal interface and the activity of trophoblasts. Of course, this needs to be based on more researches.

\section{Conclusion}

In conclusion, we found that G-CSF was mainly expressed in villi during early pregnancy, and the expression of G-CSF was decreased in villi of patients with RSA. The absence of G-CSF weakens the immune suppression of trophoblasts against macrophages, and the function of trophoblasts is impaired, which may be a key factor in the occurrence of RSA. In addition, G-CSF decreased the rate of abortion in RSA mice, and might provide some assistance in the treatment of patients with RSA.

\section{Materials And Methods}

\section{Tissue samples}

The study population was from Tongji Hospital, Huazhong University of Science and Technology, China. Included were 11 women with an uncomplicated first-term pregnancy and 10 pregnant women diagnosed with RSA. The inclusion criteria for both groups were as follows: The couples and embryos had normal chromosomes and no family genetic history, absence of anatomical deformity of reproductive organs, normal endocrine profile, normal coagulation and autoimmune function, and no vaginal infections. No women received any treatment that affected the results of the above examinations in the past 3 months before being enrolled in the study. The diagnostic criteria for RSA was two or more consecutive spontaneous abortions with the same sexual partner. There was no statistical difference in ages, gestational ages, and menstrual cycle between the two groups. The protocol was approved by the Ethics Committee of Tongji Medical College of Huazhong University of Science and Technology (2020-S150), and the informed consent of all women was obtained.

Villous and decidual tissues were collected during uterine curettage. The collected samples were washed immediately with normal saline and divided into two parts: one was fixed with polyformaldehyde for paraffin embedding and the other was stored at $-80^{\circ} \mathrm{C}$.

\section{Cell culture}

The trophoblast cell line HTR-8/SVneo was cultured in complete Dulbecco's Modified Eagle Medium (DMEM) with $10 \%$ foetal bovine serum (FBS) and 1\% penicillin/streptomycin in a $5 \% \mathrm{CO}_{2}$ incubator at $37^{\circ} \mathrm{C}$. 
The macrophage cell line RAW264.7 was cultured in DMEM with 10\% FBS, but no antibiotics, which might cause macrophage polarization. The cell was also placed in the cell incubator at $37^{\circ} \mathrm{C}$ with $5 \% \mathrm{CO}_{2}$ in humidified air.

\section{siRNA transfection}

The HTR-8/SVneo Cells were seeded in a six-well plate with a density of $40 \%-60 \%$ for transfection. Transfection was performed using lipo3000 reagent (Invitrogen) in OPTI-MEM medium (Gibco) and siRNA (RiboBio), targeting the coding region of G-CSF (50nM), and negative control siRNA (50nM), according to the manufacturer's instructions. After 48-72 hours of culture, the cells were collected for detection.

\section{Exosomes isolation}

The HTR-8/SVneo cells were planted in $10 \mathrm{~cm}$ petri dishes, cultured and expanded in large quantities, and the culture medium was collected. The medium used to extract exosome had been supercentrifuged overnight to remove its own exosome before cell culture. The medium was centrifuged at $300 \mathrm{~g}$ for 10 minutes, $2000 \mathrm{~g}$ for 30 minutes, and $12000 \mathrm{~g}$ for 45 minutes to remove whole cells and debris. The resultant supernatant was passed through a $0.22 \mu \mathrm{m}$ sterile filter and then ultracentrifuged at $120000 \mathrm{~g}$ for 2 hours. The exosome-containing pellets were washed in PBS, and ultracentrifuged again at $120000 \mathrm{~g}$ for 2 hours [33]. The final precipitates were dissolved in PBS and stored at $-80^{\circ} \mathrm{C}$. The size and morphology of exosomes were identified by TEM and nanoparticle tracking analysis NTA.

\section{Macrophage phagocytosis assay}

The exosomes were incubated with PKH26 dye (Sigma), and the staining was stopped by serum, followed by ultracentrifuged at $120000 \mathrm{~g}$ for 2 hours. The obtained exosomes were added to the RAW264.7 culture medium at a concentration of $10 \mu \mathrm{g} / \mathrm{mL}$. After 24 hours of culture, the macrophages were fixed with paraformaldehyde, stained with DAPI, sealed with anti-fluorescence quenching agent, and observed under fluorescence microscope and laser scanning confocal microscope.

\section{Animals}

Female CBA/J mice, male DBA/2J, and male BALB/c mice ( 6 weeks old) were purchased from Wuhan SLB Biotech CO., LTD. All animal studies were performed in the Laboratory Animal Centre, Tongji Hospital with all experimental protocols approved by the Animal Ethics Committee of Tongji Hospital (TJH202009005). Mice were reared under controlled temperature $\left(21^{\circ} \mathrm{C}-22^{\circ} \mathrm{C}\right)$, humidity, and 12 hours of a light/dark cycle with water and food ad libitum for 2 weeks. Female and male mice were mated 2:1 at 20:00 and checked at 6:00 the next day for vaginal plugs. The presence of a vaginal plug was designated as day 0.5 of pregnancy. Pregnant mice received daily intraperitoneal injections of recombinant G-CSF or saline from day 4.5 to 13.5. CBA/J females that mated with BALB/c were considered as Normal group and received $200 \mu \mathrm{l}$ saline $(n=6)$, and CBA/J females that mated with DBA/2 were randomized and divided into the following groups: RSA group received $200 \mu$ saline $(n=6)$ and TREAT group received $200 \mu \mathrm{l}$ of $50 \mu \mathrm{g} / \mathrm{kg}$ recombinant G-CSF [34-36]. Pregnant females were killed at day 14.5 , the total number 
of resorbing and healthy embryos were recorded, the foetal and placenta weight were also determined, and the placentas were fixed with polyformaldehyde for paraffin embedding. Abortion sites were identified by their small size accompanied by a necrotic, haemorrhagic appearance compared to normal embryos and placentas. The resorption rate was calculated as the number of total abortion/number of total implantation $\times 100 \%$.

\section{Immunohistochemistry}

Patients and mice paraffin sections were deparaffinised and rehydrated. Slides were then incubated in citric buffer for antigen retrieval. To suppress endogenous peroxidase activity, the sections were treated with $3 \%$ hydrogen peroxide. After incubation with $10 \%$ donkey serum, the primary rabbit antibodies (GCSF, abcam, diluted 1:200; G-CSFR, GeneTex, diluted 1:200; NLRP3, abcam, diluted 1:200) were applied overnight at $4^{\circ} \mathrm{C}$. The second goat-anti-rabbit antibody was incubated after washing with PBS for three times. DAB was used as a chromogen, and a light counterstaining was performed with haematoxylin. After dehydrating and drying, the sections were sealed with neutral resin and observed under an optical microscope. The images were analysed semi-quantitatively by imageJ software, and MOD = intDen/Area.

\section{RNA isolation and quantitative real-time polymerase chain reaction (RTPCR)}

Total RNA was extracted by TRIzol reagent from tissues or cells. Two $\mu \mathrm{g}$ RNA of every sample was converted to CDNA by reverse transcription. Quantitative real-time PCR analysis of genes was done using primers described in an additional file (Additional file 6: Table S1) and detected by the CFX96TM real-time system. Gene expression data were normalized to the mRNA levels of housekeeping gene GAPDH.

\section{Western blot}

Total proteins were lysed by RIPA buffer from tissues or cells. Twenty $\mu$ g proteins were loaded on a $10 \%$ Bis-Tris SDS gel and run for approximately 2 hours at $120 \mathrm{~V}$, and then transferred to the PVDF membranes. Membranes were then blocked in $5 \%$ BSA and immunoblotted with primary antibodies (GCSF, abcam, diluted 1:1000; G-CSFR, GeneTex, diluted 1:1000; CD9, abcam, diluted 1:1000; TSG101, abcam, diluted 1:1000; calnexin, abcam, diluted 1:1000; NLRP3, abcam, diluted 1:1000; GAPDH, abcam, diluted 1:1000) overnight at $4^{\circ} \mathrm{C}$. Membranes were washed with TBST and incubated in the goat antirabbit or anti-mouse secondary antibodies (Servicebio, diluted 1:5000) for 1 hour at room temperature. After three washes with TBST, the membranes were coloured by an ECL system. The images were analysed semi-quantitatively by image $J$ software, and the protein expression data were normalized to the levels of GAPDH.

\section{Migration assay}

Cell migration was assayed using a Transwell chamber. Cells suspended in serum-free media were seeded in the upper chambers at a density of $2 \times 10^{4}$ cells per well, and $500 \mu \mathrm{L}$ complete DMEM with $20 \%$ FBS was added to the lower chambers. After 24 hours of culture, cells in the upper chamber were removed with a cotton swab, and cells on the lower surface were fixed and stained with purple crystal. 
Excised and mounted filter membranes were photographed using an optical microscope, and cells in four random fields per sample were counted and recorded.

\section{Proliferation assay}

Cell proliferation was determined by CCK8 assay. Cells were plated in 96-well plates at a density of $4 \times$ $10^{3}$ cells per well. The CCK8 mixture was prepared according to DMEM: CCK8 $=9: 1$ and added to each well at $0 \mathrm{~h}, 12 \mathrm{~h}, 24 \mathrm{~h}, 48 \mathrm{~h}$ and $72 \mathrm{~h}$ after cell intervention, respectively. Absorbance at $450 \mathrm{~nm}$ was measured using an enzyme-labelled instrument after $2 \mathrm{~h}$ of culture.

\section{Statistical analysis}

SPSS version 22.0 software was used for data analysis. Results were presented as mean \pm SEM. One-way analysis of variance (ANOVA) was used to assess statistical significance among groups and Bonferroni post hoc analysis was performed where appropriate. Differences where $p<0.05$ were considered statistically significant.

\section{Abbreviations}

RSA

Recurrent spontaneous abortion; G-CSF:Granulocyte-colony stimulating factor; G-CSFR:G-CSF receptor; TEM:transmission electron microscopy; NTA:nanoparticle tracking analysis.

\section{Declarations}

\section{Acknowledgments}

Not applicable.

\section{Authors' contributions}

$L F, H L$ and $P G$ conceived and designed the experiments; $H Z, Y J, Y C$ and $S Z$ performed the experiments; $P G, L W, Y Z, X Z$ and $J Z$ analysed and interpreted the results of experiments; PG, JL and XG drafted the manuscript; LF, JY and SW reviewed and edited the manuscript. All authors read and approved the final manuscript.

\section{Funding}

This project was supported by the National Key Research and Development Program of China and the Health Commission of Hubei Province scientific research project (No.2018YFC1002903 and No.WJ2021M129)

\section{Availability of data and materials}

All data generated or analysed during this study are included in this published article. 


\section{Ethics approval and consent to participate}

The study was approved by the Ethics Committee of Tongji Medical College of Huazhong University of Science and Technology (2020-S150) and the Animal Ethics Committee of Tongji Hospital (TJH202009005).

\section{Consent for publication}

All authors consent for publication.

\section{Competing interests}

The authors declare no conflict of interest.

\section{References}

1. Pfeifer S, Goldberg J, Lobo R, Thomas M, Widra E, Licht M, et al. Definitions of infertility and recurrent pregnancy loss: a committee opinion. Fertil Steril. 2013;99(1):63.

2. Garrido-Gimenez C, Alijotas-Reig J. Recurrent miscarriage: causes, evaluation and management. Postgrad Med J. 2015;91(1073):151-62.

3. Zhu XX, Yin XQ, Hei GZ, Wei R, Guo Q, Zhao L, et al. Increased mir-6875-5p inhibits plasmacytoid dendritic cell differentiation via the stat3/e2-2 pathway in recurrent spontaneous abortion. Mol Hum Reprod. 2021.

4. Sugiura-Ogasawara M, Ozaki Y, Suzumori N. Management of recurrent miscarriage. J Obstet Gynaecol Re. 2014;40(5):1174-9.

5. Grandone E, Tiscia GL, Mastroianno M, Larciprete G, Kovac M, Tamborini Permunian E, et al. Findings from a multicentre, observational study on reproductive outcomes in women with unexplained recurrent pregnancy loss: the ottilia registry. Hum Reprod. 2021;36(8):2083-90.

6. Gao P, Zha Y, Gong X, Qiao F, Liu H. The role of maternal-foetal interface inflammation mediated by NLRP3 inflammasome in the pathogenesis of recurrent spontaneous abortion. Placenta. 2020;101:221-9.

7. Ding J, Zhang Y, Cai X, Zhang Y, Yan S, Wang J, et al. Extracellular vesicles derived from $\mathrm{m} 1$ macrophages deliver mir-146a-5p and mir-146b-5p to suppress trophoblast migration and invasion by targeting traf 6 in recurrent spontaneous abortion. Theranostics. 2021;11(12):5813-30.

8. Tian F, Qin C, Li X, Wu F, Liu X, Xu W, et al. Decreased stathmin-1 expression inhibits trophoblast proliferation and invasion and is associated with recurrent miscarriage. Am J Pathol. 2015;185(10):2709-21.

9. Ding J, Cheng Y, Zhang Y, Liao S, Yin T, Yang J. The mir-27a-3p/usp25 axis participates in the pathogenesis of recurrent miscarriage by inhibiting trophoblast migration and invasion. J Cell Physiol. 2019;234(11):19951-63. 
10. Aldo PB, Racicot K, Craviero V, Guller S, Romero R, Mor G. Trophoblast induces monocyte differentiation into cd14+/cd16 + macrophages. Am J Reprod Immunol. 2014;72(3):270-84.

11. Wang XQ, Zhou WJ, Hou XX, Fu Q, Li DJ. Trophoblast-derived cxcl16 induces m2 macrophage polarization that in turn inactivates nk cells at the maternal-fetal interface. Cell Mol Immunol. 2018;15(12):1038-46.

12. Wang S, Sun F, Han M, Liu Y, Zou Q, Wang F, et al. Trophoblast-derived hyaluronan promotes the regulatory phenotype of decidual macrophages. Reproduction. 2019;157(2):189-98.

13. Zhang YH, Aldo P, You Y, Ding J, Kaislasuo J, Petersen JF, et al. Trophoblast-secreted soluble-pd-I1 modulates macrophage polarization and function. J Leukocyte Biol. 2020;108(3):983-98.

14. Smith A, Witte E, McGee D, Knott J, Narang K, Racicot K. Cortisol inhibits csf2 and csf3 via dna methylation and inhibits invasion in first-trimester trophoblast cells. Am J Reprod Immunol. 2017;78(5):1-14.

15. Furmento VA, Marino J, Blank VC, Roguin LP. The granulocyte colony-stimulating factor (g-csf) upregulates metalloproteinase-2 and vegf through pi3k/akt and erk $1 / 2$ activation in human trophoblast swan 71 cells. Placenta. 2014;35(11):937-46.

16. Scarpellini F, Klinger FG, Rossi G, Sbracia M. Immunohistochemical study on the expression of g-csf, $\mathrm{g}$-csfr, vegf, vegfr-1, foxp3 in first trimester trophoblast of recurrent pregnancy loss in pregnancies treated with g-csf and controls. Int J Mol Sci. 2020;21(1):285.

17. Würfel W. Treatment with granulocyte colony-stimulating factor in patients with repetitive implantation failures and/or recurrent spontaneous abortions. J Reprod Immunol. 2015;108:123-35.

18. Eapen A, Joing M, Kwon P, Tong J, Maneta E, De Santo C, et al. Recombinant human granulocytecolony stimulating factor in women with unexplained recurrent pregnancy losses: a randomized clinical trial. Hum Reprod. 2019;34(3):424-32.

19. Eftekhar M, Naghshineh E, Khani P. Role of granulocyte colony-stimulating factor in human reproduction. J Res Med Sci. 2018;23:7.

20. Covone AE, Mutton D, Johnson PM, Adinolfi M. Trophoblast cells in peripheral blood from pregnant women. Lancet. 1984;2(8407):841-3.

21. Johansen M, Redman CW, Wilkins T, Sargent IL. Trophoblast deportation in human pregnancy-its relevance for pre-eclampsia. Placenta. 1999;20(7):531-9.

22. Xiang YJ, Hou YY, Yan HL, Liu H, Ge YX, Chen N, et al. Mesenchymal stem cells-derived exosomes improve pregnancy outcome through inducing maternal tolerance to the allogeneic fetus in abortionprone mating mouse. Kaohsiung J Med Sci. 2020;36(5):363-70.

23. Atay S, Gercel-Taylor C, Suttles J, Mor G, Taylor DD. Trophoblast-derived exosomes mediate monocyte recruitment and differentiation. Am J Reprod Immunol. 2011;65(1):65-77.

24. Rodrigues C, Serrano E, Patricio MI, Val MM, Albuquerque P, Fonseca J, et al. Stroma-derived il-6, gcsf and activin-a mediated dedifferentiation of lung carcinoma cells into cancer stem cells. Sci Rep. 2018;8(1):11573. 
25. Di Nicuolo F, Specchia M, Trentavizi L, Pontecorvi A, Scambia G, Di Simone N. An emerging role of endometrial inflammasome in reproduction: new therapeutic approaches. Protein Peptide Lett. 2018;25(5):455.

26. Li Y, Zhang D, Xu L, Dong L, Zheng J, Lin Y, et al. Cell-cell contact with proinflammatory macrophages enhances the immunotherapeutic effect of mesenchymal stem cells in two abortion models. Cell Mol Immunol. 2019;16(12):908-20.

27. Wen Q, Kong Y, Zhao H, Zhang Y, Han T, Wang Y, et al. G-csf-induced macrophage polarization and mobilization may prevent acute graft-versus-host disease after allogeneic hematopoietic stem cell transplantation. Bone Marrow Transpl. 2019;54(9):1419-33.

28. D'Ippolito S, Tersigni C, Marana R, Di Nicuolo F, Gaglione R, Rossi ED, et al. Inflammosome in the human endometrium: further step in the evaluation of the "maternal side". Fertil Steril. 2016;105(1):111-8.

29. Ding J, Yang C, Zhang Y, Wang J, Zhang S, Guo D, et al. M2 macrophage-derived g-csf promotes trophoblasts emt, invasion and migration via activating pi3k/akt/erk $1 / 2$ pathway to mediate normal pregnancy. J Cell Mol Med. 2021;25(4):2136-47.

30. Gleicher N, Vidali A, Barad DH. Successful treatment of unresponsive thin endometrium. Fertil Steril. 2011;95(6):2113-23.

31. Salmassi A, Schmutzler AG, Huang L, Hedderich J, Jonat W, Mettler L. Detection of granulocyte colony-stimulating factor and its receptor in human follicular luteinized granulosa cells. Fertil Steril. 2004;81(Suppl 1):786-91.

32. Ledee N, Lombroso R, Lombardelli L, Selva J, Dubanchet S, Chaouat G, et al. Cytokines and chemokines in follicular fluids and potential of the corresponding embryo: the role of granulocyte colony-stimulating factor. Hum Reprod. 2008;23(9):2001-9.

33. Rice GE, Scholz-Romero K, Sweeney E, Peiris H, Kobayashi M, Duncombe G, et al. The effect of glucose on the release and bioactivity of exosomes from first trimester trophoblast cells. J Clin Endocrinol Metab. 2015;100(10):E1280-8.

34. Li Y, Guo R, Wang L, Li S, Zhu Z, Tu P. G-csf administration results in thrombocytopenia by inhibiting the differentiation of hematopoietic progenitors into megakaryocytes. Biochem Pharmacol. 2019;169:113624.

35. Skaznik-Wikiel ME, Sharma RK, Selesniemi K, Lee HJ, Tilly JL, Falcone T. Granulocyte colonystimulating factor in conjunction with vascular endothelial growth factor maintains primordial follicle numbers in transplanted mouse ovaries. Fertil Steril. 2011;95(4):1405-9.

36. Lee HJ, Choi CW, Kim EK, Kim HS, Kim BI, Choi JH. Granulocyte colony-stimulating factor reduces hyperoxia-induced alveolarization inhibition by increasing angiogenic factors. Neonatology. 2012;101(4):278-84.

\section{Figures}


$\mathbf{A}$

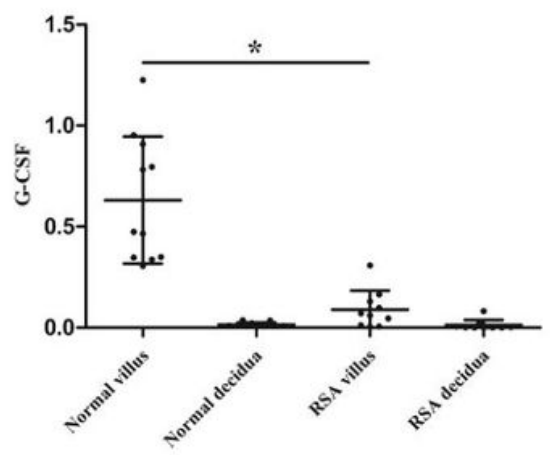

D
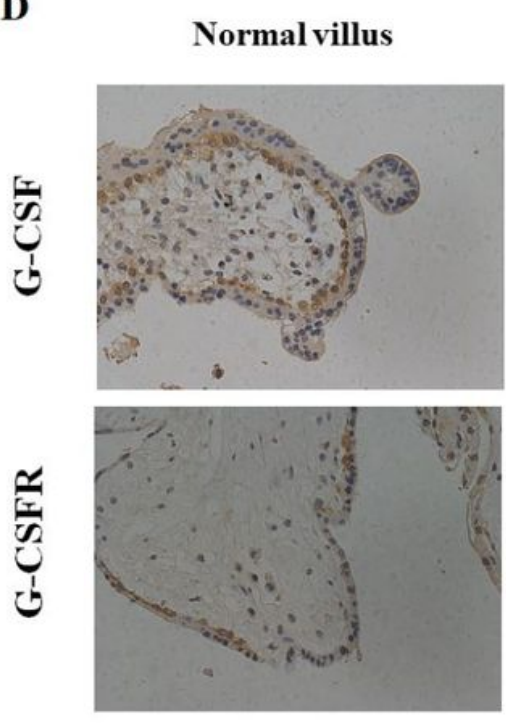

B

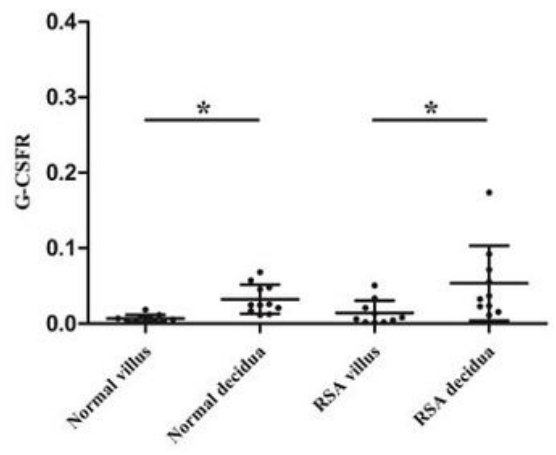

C

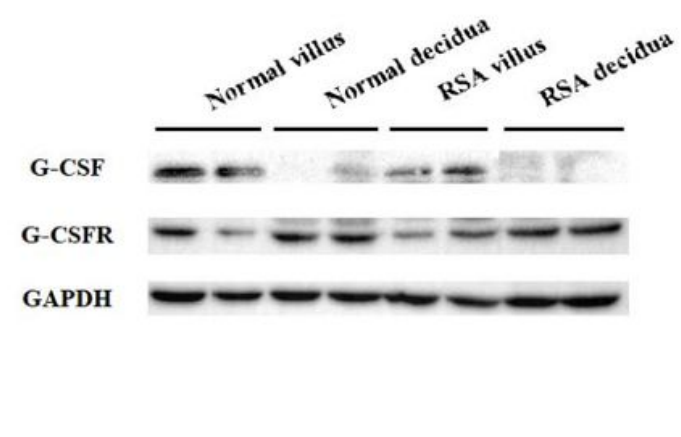

\section{Figure 1}

Normal decidua
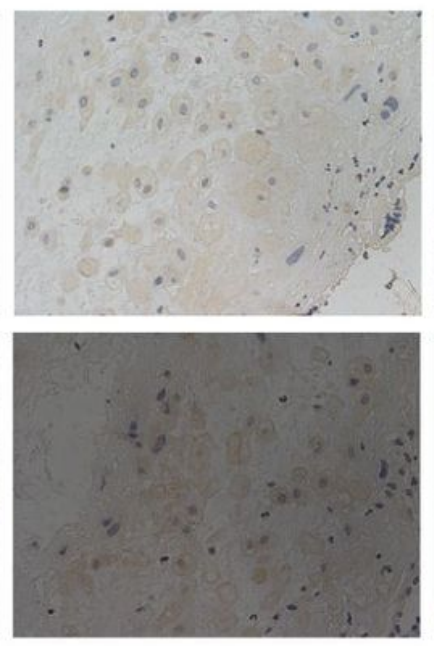

RSA villus
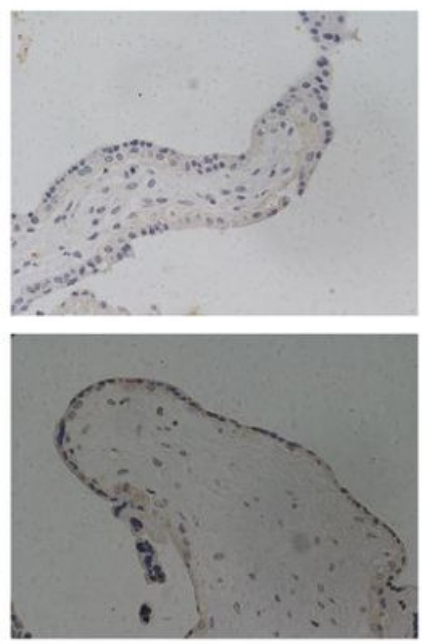

RSA decidua
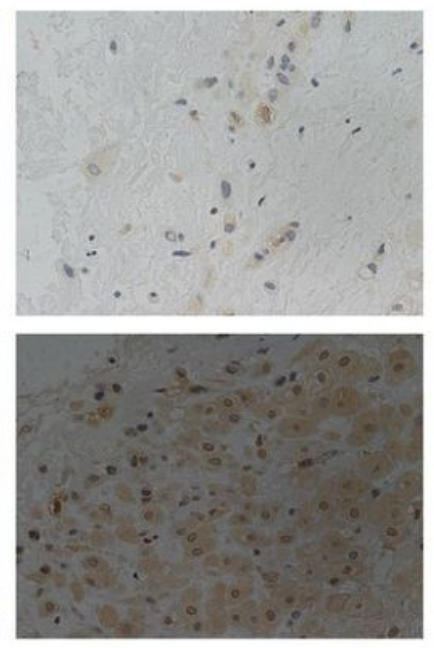

Expression of G-CSF and G-CSFR in villous and decidual tissues. Samples were detected by RTPCR (A, B, *: $P<0.05)$, western blot $(C)$ and immunohistochemistry $(D$, original magnification $\times 200)$. 
$\mathbf{A}$

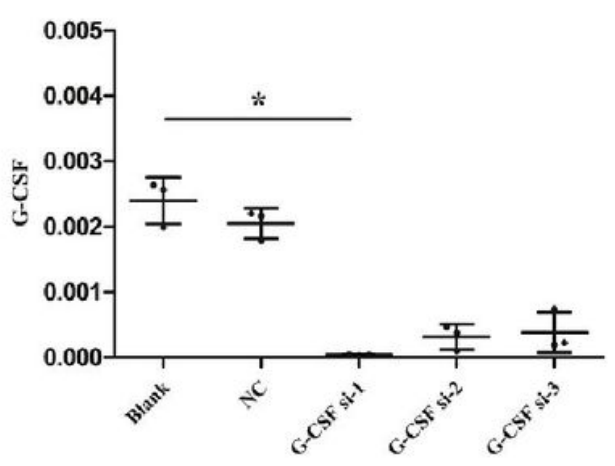

B

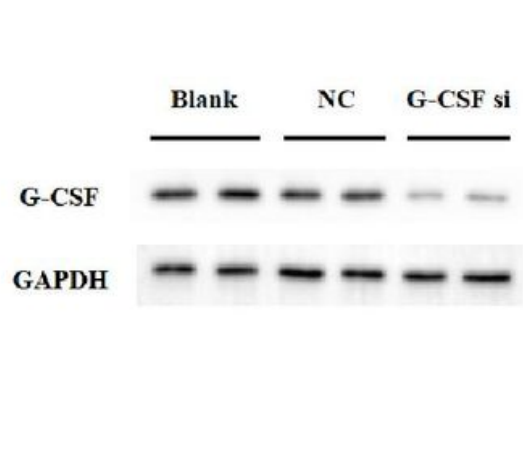

C

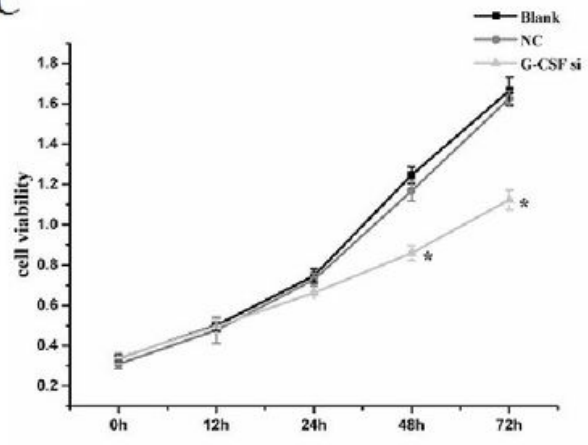

D

Blank

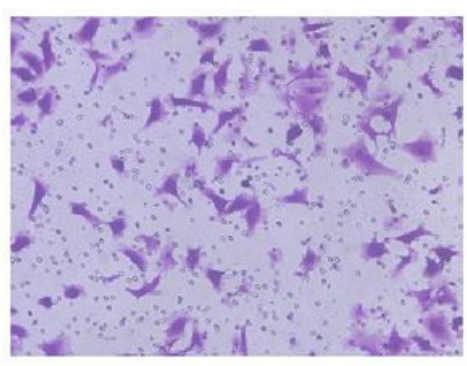

$\mathrm{NC}$

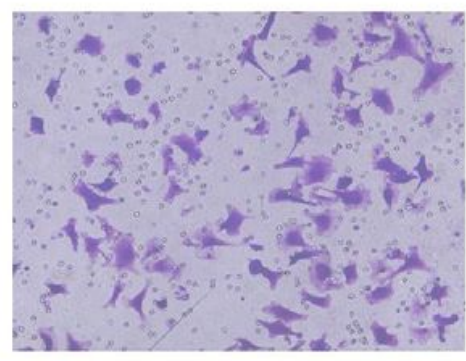

G-CSF si

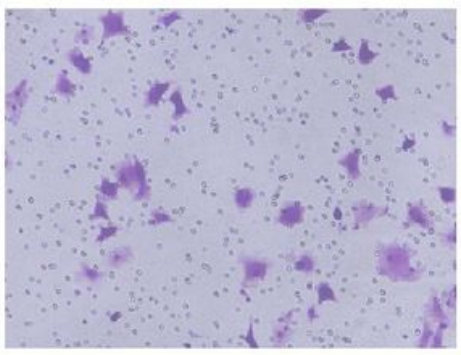

Figure 2

Effects of down-regulation of G-CSF on trophoblasts. (A) The silencing effect of three siRNAs against GCSF was detected by RTPCR, $*$ : $P<0.05$. (B) The most effective siRNA was selected and verified by Western blot. (C) Proliferative capacity of trophoblasts was evaluated by CCK8 test, *: $\mathrm{P}<0.05$ vs Blank and NC. (D) Migratory capacity of trophoblasts was evaluated by transwell test, original magnification $\times$ 100. All data was from three independent experiments. 
A

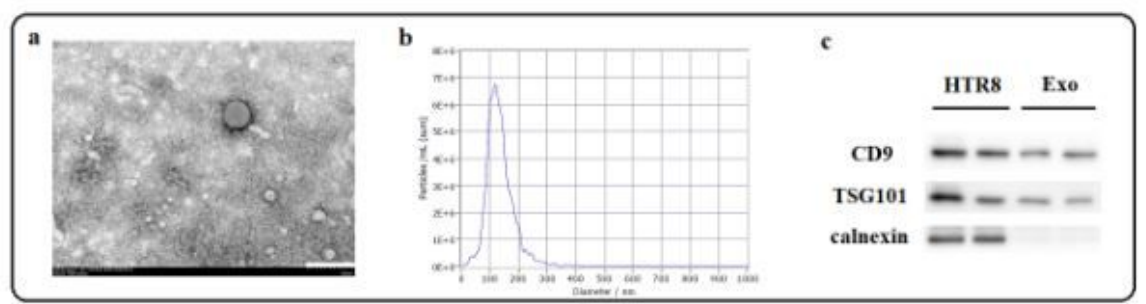

B

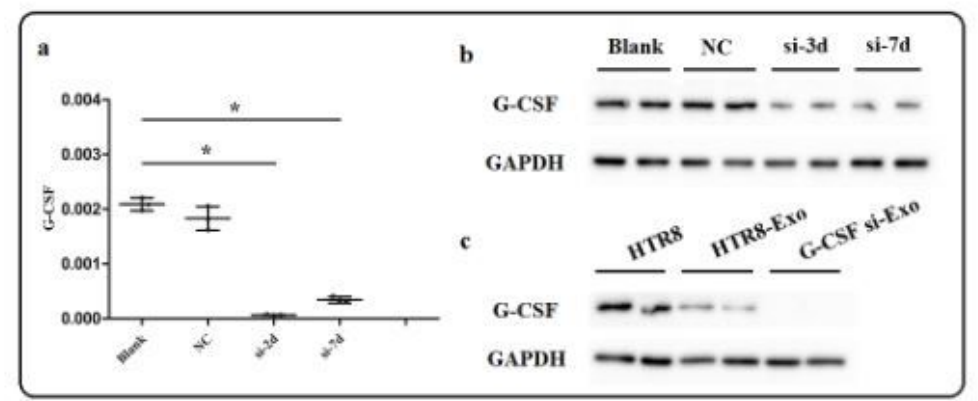

C

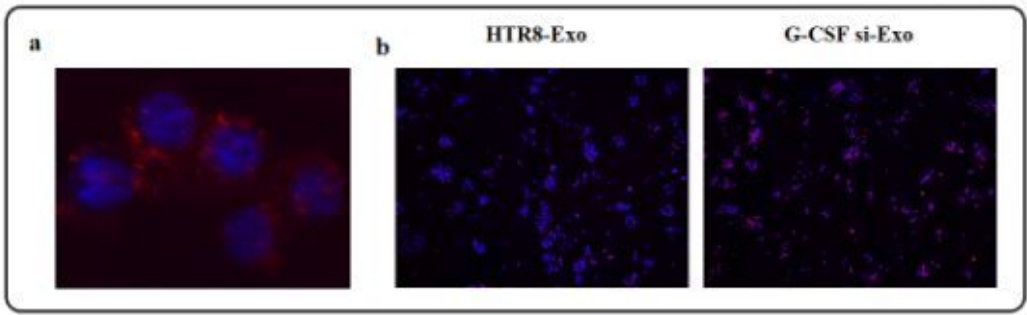

D

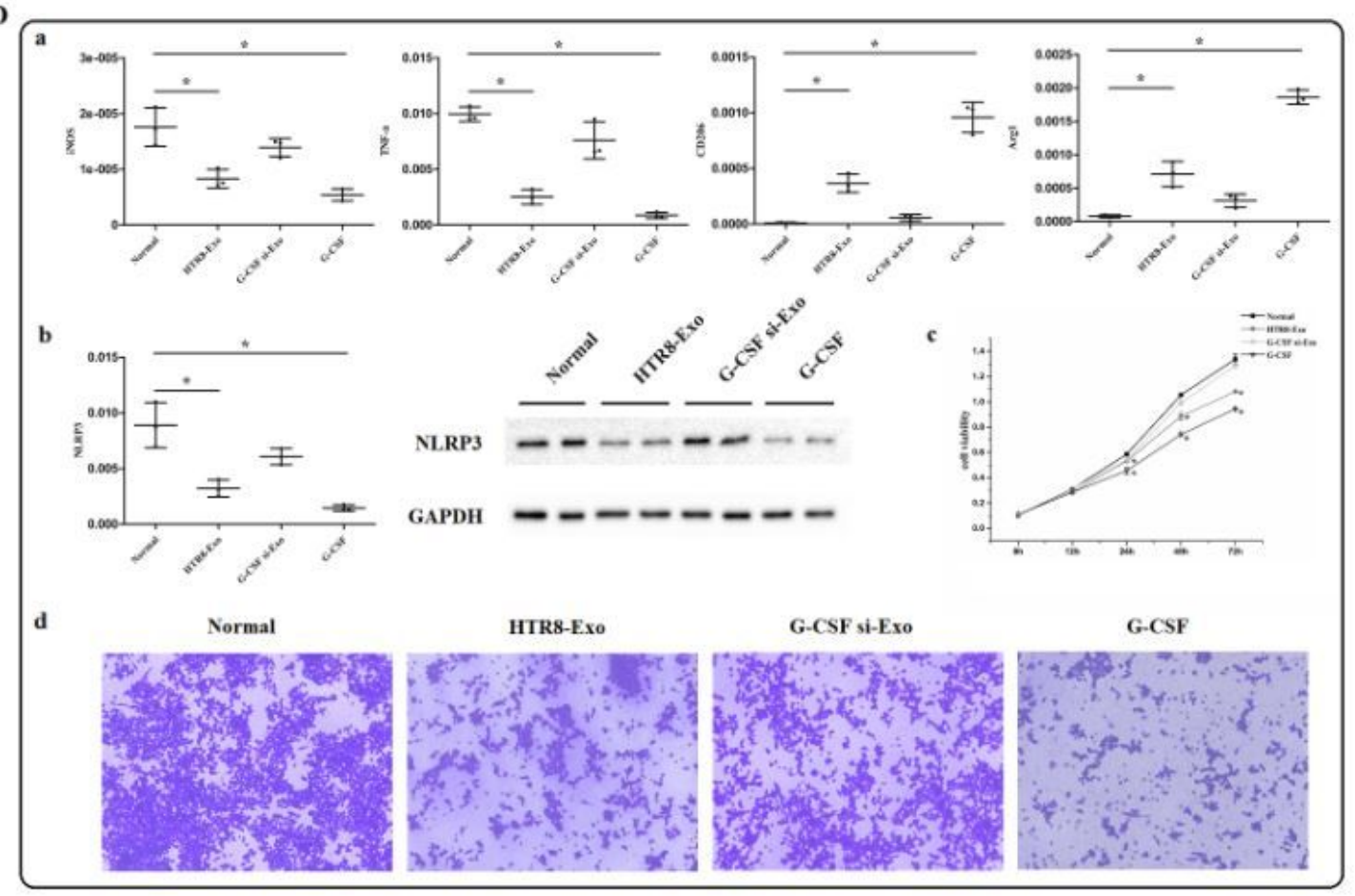

Figure 3

Role of G-CSF and Exosomes in Trophoblasts Regulation on Macrophages. (A) Exosomes derived from trophoblasts were identified by TEM (a, Scale bar: 200nm), NTA (b) and Western blot (c). (B) The expression of G-CSF in trophoblasts after siRNA transfection for 2-3 days and 7 days was detected by RTPCR (a, *: P < 0.05) and western blot (b); the expression of G-CSF in exosomes derived from normal trophoblasts and trophoblasts transfected with siRNA was detected by Western blot (c). (C) Uptake of 
exosomes by macrophages was observed by laser scanning confocal microscope (a, original magnification $\times 400$ and zoom $\times 5$ ) and fluorescence microscope $(b$, original magnification $\times 100)$. (D) Markers for macrophage type, iNOS, TNF-a, CD206 and Arg1, were detected by RTPCR $(a, *$ : $P<0.05)$; the expression of NLRP3 inflammasome in macrophages was detected by RTPCR and Western blot $(b, *: P<$ $0.05)$; proliferative capacity of macrophages was evaluated by CCK8 test $(c, *$ : $P<0.05)$; migratory capacity of macrophages was evaluated by transwell test (d, original magnification $\times 100)$. All data was from three independent experiments.

A

a

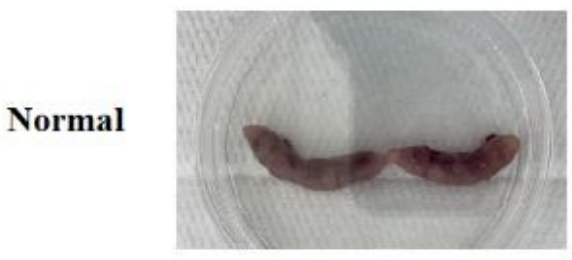

RSA

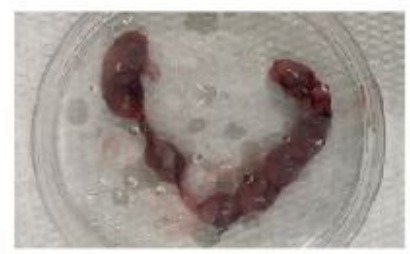

TREAT

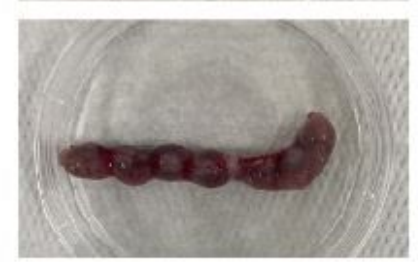

b

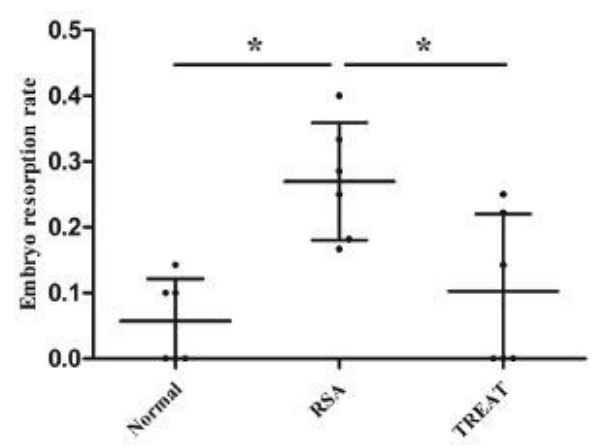

B

Normal

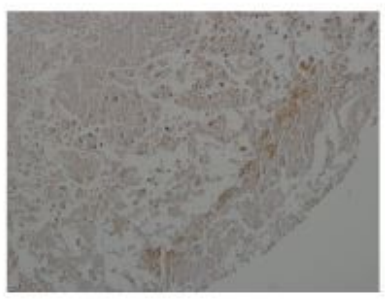

G-CSFR
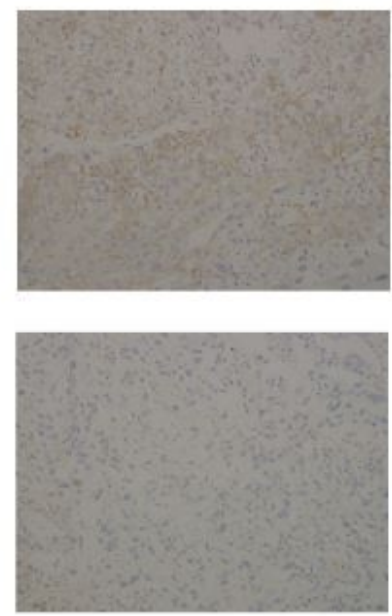

RSA
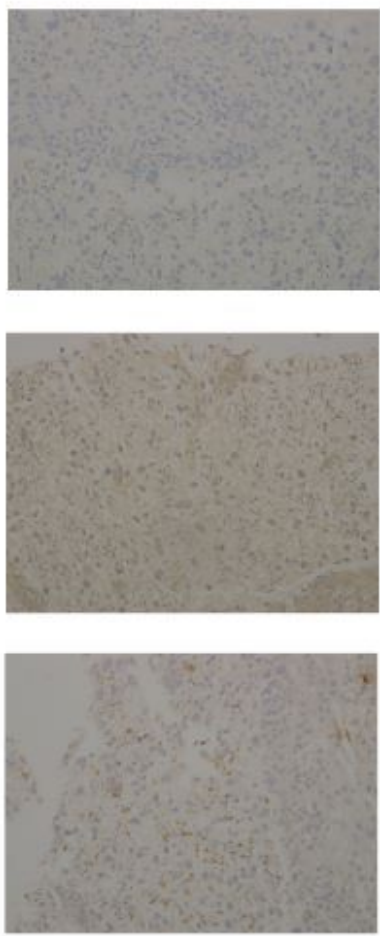

TREAT
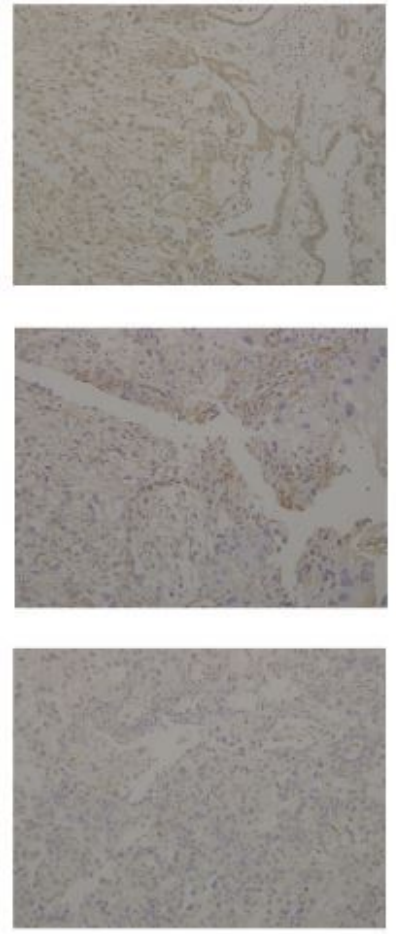


\section{Figure 4}

The pregnancy outcome of the mice model and the molecular expression at the maternal-foetal interface. (A) Representative pictures of uteri from three groups of mice (a) and embryo resorption rate of mice (b, *: $P<0.05)$. (B) Expression of G-CSF, G-CSFR and NLRP3 in placentas from three groups of mice, original magnification $\times 200$.

\section{Supplementary Files}

This is a list of supplementary files associated with this preprint. Click to download.

- Additionalfile1.FigureS1.tif

- Additionalfile2.Figures2.tif

- Additionalfile3.FigureS3.tif

- Additionalfile4.Figures4.tif

- Additionalfile5.FigureS5.tif

- Additionalfile6.Tables1.docx 